\title{
Aspirin: past, present and future
}

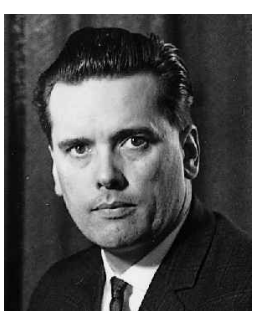

This article is based on the

Milroy Lecture given at the Royal

College of

Physicians on 26 June 2000 by

Peter C Elwood MD FRCP FFPHM,

Honorary

Professor,

University of Wales

College of

Medicine, Cardiff

Clin Med JRCPL 2001;1:132-37

$A B$
$h$
$t$
$t$
$t$

ABSTRACT - Many folk remedies used since prehistoric times have depended upon salicylates for their effect. One hundred years ago aspirin was formulated from salicylic and acetic acids. It was the first drug to be synthesised and its formulation is regarded as the foundation of the modern pharmaceutical industry.

The benefit of low-dose aspirin as a prophylactic after a thrombotic event was first reported 25 years ago. Its use after coronary or cerebral thrombosis is virtually mandatory, unless there are signs of intolerance. A 'loading dose' of soluble aspirin should be given on first contact with a patient who may be suffering from myocardial infarction. Patients considered to be at increased risk of a vascular event should also be advised to carry their own aspirin and, if they experience sudden severe chest pain, to chew and swallow a $300 \mathrm{mg}$ tablet or a soluble preparation immediately.

The current phase of the aspirin story is, however, not over, and its possible value in a variety of conditions, including dementia and certain cancers, seems likely to ensure that it will long continue to play a remarkable part in clinical practice.

\section{The medicinal use of salicylates}

Salicylates are widely distributed in plants and many herbal remedies probably depend for their effect on the presence of a salicylate. Around $400 \mathrm{BCE}$ Hippocrates recommended a brew of leaves from the willow tree (Cortex salicis), a rich source of salicylates, for the relief of pain in childbirth.

The attention of chemists seems to have been first drawn to salicylates after the Reverend Edward Stone of Chipping Norton wrote, in 1763, to the President of the Royal Society in London, the most prestigious scientific body in the world at that time ${ }^{1}$. In common with many physicians, Stone believed in the so-called Doctrine of Signatures, namely that alongside many maladies providence had provided the means for their cure. He was therefore attracted to the willow tree because it flourishes in moist and wet soils and it was in such situations that fevers were then common. Stone reported how he had used increasing doses of a powder prepared from the bark of the common white willow tree, dried 'upon the outside of a baker's oven for more than three months'. He described the effective treatment with this powder of 'fever in over 50 patients suffering from various agues'.

Salicylic acid seems to have been first synthesised from carbolic acid around 1859 by a German chemist and it came to be widely used for the relief of pain and fever. Because of its irritant action on the stomach, efforts were then made by chemists to find a form or a derivative of salicylic acid which could be better tolerated. In 1897 Felix Hoffman, a chemist working in a laboratory owned by Friedrich Bayer in Elberfeld, Germany, and stimulated by earlier work on acetylation, formulated a pure and stable form of acetyl salicylic acid simply by mixing acetic and salicylic acids ${ }^{2}$. Hoffman was motivated by the suffering of his father, who had severe arthritis, and could not tolerate salicylic acid.

Bayer gave the name A-spirin to the new preparation, from acetylation $(A-)$, together with spirin, part of the name for meadowsweet (Spiraea ulmaria), a plant rich in salicylates. Bayer patented the name and commenced to market the product in 1899 . It was a huge success and sales grew rapidly. In fact, the company set up by Friedrich Bayer \& Company is reckoned to have been the first pharmaceutical company, and the production of aspirin is generally accepted to have laid the foundation of the modern pharmaceutical industry.

Aspirin was accepted widely with great enthusiasm, and it was soon recommended in the medical press for use with fever, migraine, the pain of inoperable cancer, rheumatoid arthritis, gout, rheumatic fever, acute tonsillitis, corns and warts ${ }^{3}$. In 1997, the National Library of Medicine listed over 23,000 papers on aspirin, and it has been estimated that currently a paper on aspirin is published on average every two hours!

\section{Platelets and vascular disease}

The early microscopists saw platelets, but they dismissed them as either cell debris, or dirt on the slides. Donné, a French physiologist, seems to have been the first, in 1842 , to describe platelets ${ }^{4}$. He called them 'globulins $d u$ chyle' and believed they coalesced to form white blood cells. Osler, the Regius Professor of Physic in Oxford, recognised that platelets play a part in thrombosis, and in 1874 
he described pseudo-podia and the adherence of platelets to fibrin when stimulated ${ }^{5}$.

Nevertheless, the role of platelets and their part in thrombosis was long debated and it was not until perhaps the late 1950s that there was general agreement about their role in coronary and other thrombotic processes. Since then a vast amount of work has led to the present understanding of platelet function. Early papers of particular relevance were published in 1974 by Haerem ${ }^{6}$, who showed that tiny platelet emboli could often be found in the coronary micro-circulation in cases of sudden death in which there was no major coronary thrombus, and in 1984 by Michael Davies, who showed that a platelet plug could be found at the head of almost every thrombus in the coronary vessels ${ }^{7}$.

\section{Aspirin and platelet activity}

An effect of aspirin on platelets was first described by Morris in $1967^{\circ}$. O'Brien and others immediately took this up and showed that reductions in the dose of aspirin down to quite low levels lost none of the platelet anti-aggregant effects, and that the effect of a single dose of aspirin is detectable on platelet aggregation for about ten days - that is, until all the affected platelets have been replaced ${ }^{9,10}$.

Aspirin, even at very low doses, affects platelet function primarily by inhibiting platelet cyclo-oxygenase, an enzyme involved in the formation of the aggregating agent thromboxane $\mathrm{A}_{2}$. In fact, aspirin has other effects on haemostatic mechanisms, some of which are due to interference with vitamin $\mathrm{K}$ in the hepatic synthesis of the coagulation factors VII, IX and $\mathrm{X}^{11}$. These may add to its protective effect.

\section{Aspirin and coronary thrombosis}

The first randomised controlled trial of aspirin in the prevention of vascular events was reported in $1974^{12}$, and to date over 145 randomised controlled trials of aspirin in vascular disease have been reported in the medical press. These have involved a total of over 100,000 patients, amongst whom there were almost 11,000 heart attacks and strokes ${ }^{13}$. These trials have established aspirin, used in cardiovascular disease, as the most thoroughly tested drug and have shown it to be probably the most costeffective of all drugs used in clinical practice today.
Table 1. The reduction in vascular events by long-term lowdose aspirin prophylaxis. (Based on the combined results of 145 randomised controlled trials as reported in ref 13)

\begin{tabular}{|c|c|c|}
\hline \multicolumn{3}{|l|}{ Reduction in various clinical groups: } \\
\hline Patients with prior $\mathrm{Ml}$ & 11 trials & $25 \%$ reductio \\
\hline Patients with an acute $\mathrm{MI}$ & 9 trials & $29 \%$ reduction \\
\hline Patients with a prior stroke or TIA & 18 trials & $22 \%$ reduction \\
\hline Other high risk groups of patients & 104 trials & $32 \%$ reduction \\
\hline \multicolumn{3}{|l|}{ Reduction in the separate outcomes: } \\
\hline Reduction in non-fatal Mls & 122 & $34 \%$ \\
\hline Reductior & 124 & $25 \%$ \\
\hline Reduction in all vasc & 144 trials & $17 \%$ reduction \\
\hline Reduction in all-caus & 144 & $16 \%$ redi \\
\hline \multicolumn{3}{|c|}{ Relative reduction in different patient groups: } \\
\hline Males a & \multicolumn{2}{|c|}{ similar reduction } \\
\hline Older and younger & \multicolumn{2}{|c|}{ similar reduction } \\
\hline Hypertensive and normotensive & \multicolumn{2}{|c|}{ similar reduction } \\
\hline Diabetic and non-diabetic & \multicolumn{2}{|c|}{ similar reduction } \\
\hline
\end{tabular}

The results of these trials have been examined in a number of overviews. These show a remarkable consistency and indicate that aspirin reduces the incidence of non-fatal vascular events by between 30 and $40 \%$ and reduces deaths from all causes by around $20 \%$ (Table 1). The consistency in the results offers no evidence of any important differences in the proportionate benefit for patients with a variety of previous clinical conditions such as unstable angina, previous MI or stroke, peripheral vascular disease and artery and valve surgery (Table 2). Nor is there any evidence of significant heterogeneity in the reduction achieved by aspirin in different groups of patients such as male and female, older and younger, diabetic and non-diabetic etc.

Of course there are failures and some patients experience a vascular event despite aspirin prophylaxis. Some of these failures are undoubtedly due to poor compliance. Compliance was assessed in the Physicians' Health Study, in which aspirin was to be taken on alternate days. Subjects who took aspirin on every recommended day experienced a $51 \%$ reduction in vascular events, whereas those who took aspirin on less than half the days recommended showed only a $17 \%$ reduction ${ }^{14}$. Furthermore, an MI which occurs in a patient taking aspirin is likely to be of the small, non Q-wave variety ${ }^{15}$.

Table 2: Risks and benefits in aspirin prophylaxis. (Based on the combined results of 145 trials (ref 13 and others))

\begin{tabular}{|c|c|c|c|c|c|}
\hline In every 1,000 patients, per year: & $\begin{array}{l}\text { Prior } \\
\text { MI }\end{array}$ & $\begin{array}{l}\text { Prior } \\
\text { stroke }\end{array}$ & $\begin{array}{l}\text { First month } \\
\text { after stroke* }\end{array}$ & $\begin{array}{l}\text { Other high-risk } \\
\text { subjects }\end{array}$ & $\begin{array}{l}\text { Low risk } \\
\text { patients }\end{array}$ \\
\hline - the likely saving of clinical events & 38 & 35 & 8 & 22 & $2-3$ \\
\hline possibly due to aspirin & $0.5^{* *}$ & $0.1^{* *}$ & $2.0^{* *}$ & $0.1^{* *}$ & $0.5^{* *}$ \\
\hline
\end{tabular}

* The figures in this column are the additional savings during the first month after a stroke.

** These figures are not statistically significant but are the upper $99 \%$ confidence limits of estimates. 


\section{Aspirin and stroke}

The use of aspirin in the prevention of stroke appears to be in line with MI prevention both in the degree of protection and in the dose that is appropriate ${ }^{13,16}$.

Differential diagnosis between an ischaemic lesion and cerebral haemorrhage is of course impossible on clinical grounds alone. If computerised tomography (CT) can be performed and if this indicates an ischaemic lesion, aspirin should be given as early as possible; but if haemorrhage is demonstrated, then aspirin should be withheld during the acute phase of the lesion.

The question naturally arises as to the advisability of aspirin prophylaxis in the acute phase of a stroke if CT cannot be performed. The results of the Chinese trial (CAST) ${ }^{17}$ and the International Stroke Trial (IST) ${ }^{18}$ indicate that if aspirin is given in the acute phase it prevents $8-10$ deaths during the first month - a reduction that is 'modest but worthwhile'19.

A stroke in a patient with atrial fibrillation is likely to be embolic in origin. If such a stroke is suspected then anticoagulation, rather than aspirin, is the treatment of choice. Nevertheless, if facilities for monitoring are not adequate and it is decided not to use anticoagulants, aspirin should certainly be included in the management of patients with $\mathrm{AF}^{20}$.

\section{Aspirin prophylaxis in diabetic patients}

An analysis of diabetic patients within the ISIS-2 trial claimed that they derived no benefit from aspirin. The group which had conducted the ISIS-2 trial replied: 'Neither did patients born under the astrological signs of Gemini and Libra'! In other words, the analyses of subgroups of patients within a trial is fraught with uncertainties. In this particular case, a major overview showed no evidence of heterogeneity in the results within diabetic and non-diabetic patient groups ${ }^{13}$.

\section{Aspirin and venous thrombosis}

A recent trial based upon over 13,000 surgical patients demonstrated benefit from low-dose aspirin, with a reduction of $43 \%$ in pulmonary embolism and $36 \%$ in deep vein thrombosis ${ }^{21}$. Unfortunately this trial was not factorial and so no comparison can be made with prevention with anticoagulants.

\section{Aspirin and 'primary' prevention}

Can aspirin be used to prevent vascular events in subjects who have not already had an MI, stroke or other vascular event, that is, so-called primary prevention?

The trial of 22,071 American physicians who were given $325 \mathrm{mg}$ aspirin on alternate days showed a relative reduction by aspirin of $44 \%$ in the incidence of non-fatal myocardial infarction $^{22}$. On the other hand, 5,139 healthy British doctors, half of whom were given $500 \mathrm{mg}$ aspirin per day for six years, gave no evidence of protection ${ }^{23}$. Subsequent analyses of the complete results from both trials indicate a significant reduction in non- fatal infarction by aspirin of $33 \%$ and a non-significant reduction of all vascular deaths of $10 \%{ }^{13,22}$.

The Thrombosis Prevention Trial ${ }^{24}$ examined this situation further in over 5,000 men who had not had a vascular event but were judged clinically to be at increased risk of infarction. A relative reduction by aspirin of $32 \%$ non-fatal events and $20 \%$ deaths was found. A Swedish trial based upon patients with angina pectoris ${ }^{25}$ showed a relative reduction of $34 \%$ by aspirin in men who had not had a vascular event.

The terms 'primary' and 'secondary' relate to the past history of a patient, and not to future risk. They are therefore misleading and the concept of 'primary' prevention is not necessarily in the best interests of patients. In relation to the risk of a future event, subjects simply form a continuum ranging from those at exceedingly low risk, such as younger healthy subjects, to patients who have recently had an MI. Between these extremes are subjects at risk because of age, smoking, raised cholesterol, hypertension etc. Figure 1 displays this continuum. Aspirin reduces the risk of a future vascular event by about one third right across the continuum. On the other hand, the average risk of undesirable side effects is constant in all subjects, whatever their vascular risk. Clearly, the balance between reduction of events and undesirable side effects is highly in favour of aspirin prophylaxis at the high-risk end of the continuum, but whether or not aspirin is recommended at lower levels of risk is a clinical decision, taking into account the likely risk of a future vascular event and the likely occurrence of serious side effects from the aspirin. The occurrence of a past event is only one element in this judgement.

Recently a re-analysis of sub-groups of patients within The Thrombosis Prevention Trial ${ }^{24}$ suggested that those with raised blood pressure may not benefit from low-dose aspirin. As the authors admit, however, results from post hoc subgroup analyses are highly suspect and this result is best regarded as a hypothesis requiring testing, rather than a guide for clinical practice.

\section{'Early' and 'immediate' aspirin}

'Early' aspirin: that is, aspirin given by a doctor or paramedic on first contact with a patient who has chest pain and is judged possibly to be experiencing an MI, is now accepted clinical practice in most Western countries.

- An extension of this measure would be for patients who are judged to be at high risk of infarction to be advised to carry their own aspirin and chew and swallow one or two tablets immediately they experience severe chest pain. This has been recommended ${ }^{26}$ and would certainly seem to represent a reasonable and simple extension of present practice for a number of reasons:

- Mortality during and immediately after infarction is high and rapidly decreases with time. The earlier aspirin is given the greater the savings in death and disability are likely to be.

- Early aspirin may limit the growth of a developing thrombus. Furthermore, platelet emboli have been found within the coronary circulation in subjects who have died 
suddenly ${ }^{6}$ and it is possible that aspirin, taken very early, may lead to the disaggregation of these emboli.

- The occurrence of myocardial infarction peaks between about 04.00 and 10.00 hours and this coincides with an increased sensitivity of platelets to aggregating agents ${ }^{27}$. The readiness with which a patient is likely to call a doctor, and the availability of help, is probably less during these hours than later in the day. It is also of interest that in the US Physicians' Trial it was found that the reduction in myocardial infarction by aspirin was significantly greater during the early morning (59\%) than during the rest of the day $(34 \%)^{28}$.

- Most patients in whom an infarct is proven will go on to receive thrombolytic therapy. There is a marked increase in platelet activity after thrombolysis followed by an excess in re-infarction. This is abolished by prior treatment with $\operatorname{aspirin}^{29}$.

If therefore a person is judged to be at increased risk because of raised levels of any of the risk factors for cardiovascular disease - age, smoking, blood pressure, cholesterol level etc - then advice about 'immediate' aspirin should be considered; a 'loading dose' of 300-600mg of soluble aspirin has been recommended $^{30}$. Even patients who are already taking daily low-dose aspirin should be advised to carry a few tablets of soluble aspirin. The half-life of aspirin in the circulation is only about 30 minutes and if a thrombus develops despite daily exposure to aspirin, then some sensitive platelets which have not been acted upon by aspirin have probably come into the circulation. An extra flush of $300 \mathrm{mg}$ aspirin taken in addition to a daily dose of perhaps $100 \mathrm{mg}$ could therefore be life saving and is unlikely to do any harm, as such patients will have already been screened for intolerance to aspirin.

\section{The dose of aspirin for prophylaxis}

There is little evidence that the degree of protection in long-term prophylaxis is any different with doses between about 75 and 300mg. The WHO recommendation for $100 \mathrm{mg}$ daily is therefore most reasonable.

The formulation of aspirin seems to matter little. There is however evidence that the absorption from enteric coated tablets may be low if taken with food. Furthermore, absorption is not only greatly delayed but some elderly subjects may absorb very little of the drug from such preparations. At the same time, some large and successful trials have been based on these formulations and they are clearly effective in the majority of subjects.

The aim of 'early' and 'immediate' aspirin is to get the drug into the circulation as quickly as possible. Soluble aspirin, and at least $300 \mathrm{mg}$, should therefore be used $^{30}$. Significant quantities of aspirin have been detected in the plasma within a few minutes of the ingestion of soluble aspirin, together with a total inhi- bition of aggregation, again within minutes ${ }^{31}$. If a soluble preparation is not available, one or two tablets of standard aspirin should be chewed and swallowed; but clearly, coated tablets are unsuitable for immediate or early use.

\section{Side-effects of low-dose aspirin}

Gastric irritation and increased occult blood are dose related and while evidence from trials suggests that the relative risks of gastric irritation and intestinal bleeding are increased by 50 to $100 \%$, the absolute risk of these symptoms with continuing lowdose aspirin is only a little higher than that which occurs with placebo tablets. The excess of clinically significant blood loss is probably only one or two in every hundred subjects on low-dose prophylaxis.

Concerns have been expressed regarding the increased risk of cerebral haemorrhage - in particular, during aspirin prophylaxis following stroke. Overviews of trials show, however, that the number of cerebral bleeding events that might be attributed to aspirin is very small (and despite the huge numbers in the overviews, the excess is technically not statistically significant). Even in the acute phase of a stroke, the absolute number of bleeds is very considerably exceeded by the numbers of events prevented by aspirin $^{19}$.

\section{Relative and absolute reductions}

The relative, or proportionate reduction of events in aspirin trials conducted in post MI patients is about one third: that is, a reduction in incidence from perhaps $12 \%$ in the year following infarction to perhaps $8 \%$. The absolute number of events prevented, however, is only around 4 per year in every hundred, while the other 96 patients are exposed to the costs and the side

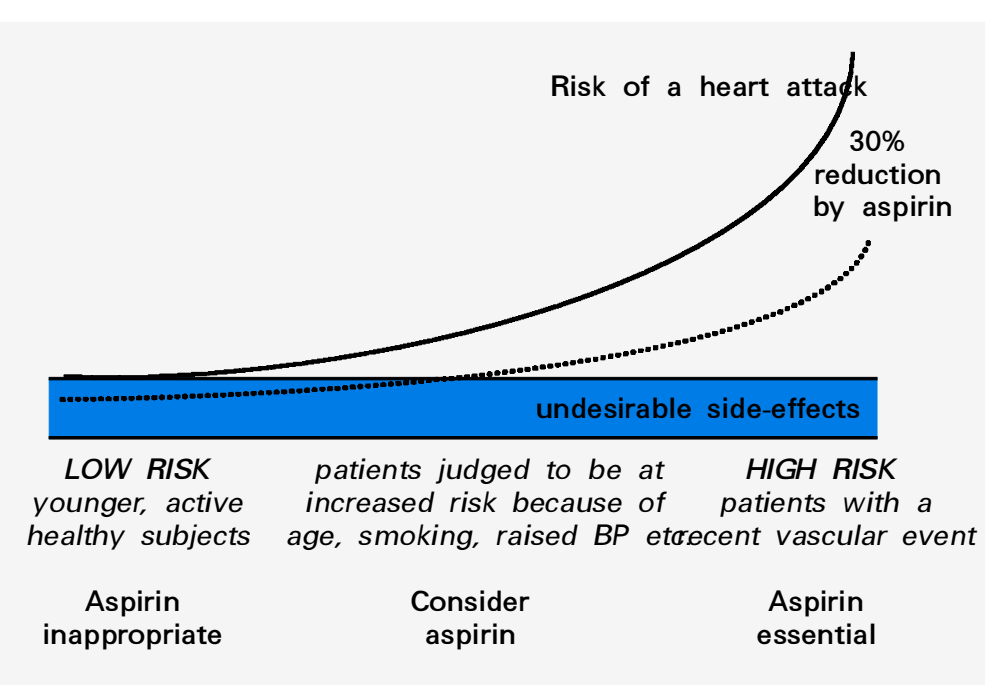

Fig 1. Aspirin and the reduction of risk of vascular disease events. (Based on the combined results of 145 randomised controlled trials as reported in ref 13 . 
effects of the aspirin. While the relative reduction in incident events appears to be very closely similar in subjects at all levels of risk, the absolute reduction in these will depend upon the basic risk of a vascular event within each group, and the following general conclusions seem appropriate:

- of every thousand post-MI or post-stroke patients put on aspirin, about 40 will avoid a thrombotic event each year. One or two may experience a serious bleed requiring transfusion. Perhaps about 5\% will experience minor side effects.

- of every thousand subjects with evidence of increased cardiovascular risk (older and/or smoking and/or raised blood pressure and/or raised cholesterol level etc), perhaps 10 to 30 subjects, depending on their level of risk, will avoid a thrombosis if put on aspirin, while one or two may experience a serious bleed, requiring transfusion.

- of every thousand healthy subjects, with no clinical evidence of increased cardiovascular risk, perhaps one or two will avoid a thrombosis if put on aspirin, while one or two may experience a serious bleed.

\section{Alternatives to aspirin}

Low-dose aspirin is inappropriate for about $8-10 \%$ of subjects. If it causes gastric irritation or other mild symptoms entericcoated tablets should be tried. However, it is contra-indicated if there is a history of sensitivity or peptic ulceration and in such cases an alternative to aspirin should be considered. These are all much more expensive, and are not without side effects.

- Dipyridamole appears to give no additional benefit if given along with aspirin, and is an inadequate substitute for $\operatorname{aspirin}^{32}$.

- Ticlopidine: a reduction in vascular events similar in magnitude to aspirin has been demonstrated, but the drug causes severe neutropenia in almost $1 \%$ of patients ${ }^{33}$. It should therefore only be used if the facilities for white cell monitoring are adequate.

- Clopidogrel (Plavix): this derivative of ticlopidine gives just about the same benefit as aspirin ${ }^{34}$. It is therefore best reserved for use as an alternative to aspirin in the few patients who cannot tolerate aspirin in any formulation.

- Anticoagulants: an overview of 26 RCTs with 73,000 subjects indicates protection of similar magnitude as aspirin, but an increased number of serious bleeding episodes. The general conclusion by the authors is that the addition of anticoagulants to aspirin is not justified ${ }^{35}$.

- IIb IIIa inhibitors: these have been shown to be of benefit when added to aspirin, but they are as yet only suitable for use in the acute phase of infarction ${ }^{36}$.

\section{Cost/effectiveness of aspirin}

When used to reduce cardiovascular risk, aspirin is the most cost-effective of all drugs available at present in clinical practice.
Key Points

The synthesis of aspirin, 100 years ago, was the foundation of the modern pharmaceutical industry

Twenty five years ago aspirin was first shown in an RCT to reduce the risk of death following myocardial infarction. It is now the most thoroughly tested and most highly cost-effective drug used in clinical practice

Growing evidence indicates that it is likely to have many other uses, including a reduction in cognitive decline and dementia, colon and other cancers

During the year or two after a myocardial infarction or a stroke, the cost of treating about 40 patients to prevent one vascular event each year is only about $\mathfrak{E} 80$. This contrasts somewhat with around $\mathfrak{E} 8,500$ for each event prevented by clopidogrel, and around $\mathfrak{E} 12-17,500$ for cholesterol lowering with a statin ${ }^{37}$.

It must however be emphasised that aspirin is not in competition with other drugs, nor is it an alternative to other therapies. Thus hypertension, raised cholesterol level etc should be treated, but in these and any situations in which there is evidence of an increased risk of a vascular event, long-term low-dose aspirin prophylaxis should also be given, unless contra-indicated.

\section{The future of aspirin}

When used in vascular disease aspirin has become the victim of its own success. The cost benefit of aspirin prophylaxis is so well established and so attractive that further placebo-controlled trials are neither acceptable nor necessary in vascular disease. At the same time, concern has repeatedly been expressed that, despite convincing evidence of its effectiveness, the knowledge of the benefits of aspirin and its use by doctors is much less than desirable. Everything possible should therefore be done to promote aspirin prophylaxis in appropriate subjects, and to achieve a high level of compliance in tablet taking ${ }^{14}$.

Perhaps the most hopeful new use of aspirin is in cognitive decline and dementia. The most obvious cause of decline of cognition is a clinical stroke, but the less dramatic, and often undetected, process of 'multi-infarct dementia' is now well recognised. A reduction in cognitive decline through the prevention of these multi-infarct lesions by aspirin is therefore a reasonable expectation ${ }^{38}$.

An anti-inflammatory effect of low-dose aspirin has been reported and this gives a possible biological basis for claims that anti-inflammatory drugs, including aspirin, may reduce progression in Alzheimer's disease. A number of observational studies, and one overview ${ }^{39}$, give supportive evidence.

Perhaps the most exciting possible new use of aspirin is in colon and other cancers. Several major cohort studies and smaller studies have reported a substantial reduction in colon and other gastro-intestinal cancers in habitual aspirin takers ${ }^{40}$. A number of randomised controlled trials have been set up to examine this further. 


\section{References}

1 Stone E. An account of the success of the bark of the willow in the cure of agues. Philos Trans 1763;53:195-200.

2 Kroneberg HG. Historical remarks on acetyl salicylic acid. In: Lee M (ed). 1982 Seoul International Aspirin Symposium Proceedings. Seoul: Dong Bu I-chon Dong, 1982:5-17.

3 Wilthauer J, Wohlgemut J. Uber aspirine (acetylsalicylsaure). Ther Mh (Halbmh) 1899;13:276.

4 Donné A. De l'origine des globules du sang, de leur mode de formation et de leur fin. Compt Acad Sci 1842;14:366-8.

5 Osler W. An account of certain organisms occurring in the liquor sanguinis. Proc R Soc Lond 1874;5:692-734.

6 Haerem JW. Platelet aggregates and mural microthrombin in the early stages of acute phase of coronary disease. Thromb Res 1974;5:243.

7 Davies MJ, Thomas AC. Thrombosis and acute coronary-artery lesions in sudden cardiac ischaemic death. N Eng J Med 1984;10:1137-40.

8 Morris CDW. Acetylsalicylic acid and platelet stickiness. Lancet 1967;I:279-80.

9 O'Brien JR. Effects of salicylates on human platelets. Lancet 1968;I:779-83.

10 Stuart RK. Platelet function studies in human beings receiving $300 \mathrm{mg}$ of aspirin per day. J Lab Clin Med 1970;75:463-71.

11 Rocca B, Fitzgerald GA. Erythrocytes modulate platelet function. Should we rethink the way we give aspirin? Circulation 1997;95:11-13.

12 Elwood PC, Cochrane AL, Burr ML, Sweetnam PM, et al. A randomised, controlled trial of acetyl salicylic acid in the secondary prevention of mortality from myocardial infarction. $\mathrm{Br}$ Med J 1974; I:436-40.

13 Antiplatelet Trialists' Collaboration. Collaborative overview of random trials of anti-platelet therapy - I: Prevention of death, myocardial infarction and stroke by prolonged anti-platelet therapy in various categories of patients. Br Med J 1994;308:81-106.

14 Glynn RJ, Buring JE, Manson JE, LaMotte F, et al. Adherence to aspirin in the prevention of myocardial infarction: The Physicians' Health Study. Arch Intern Med 1994;154:2649-57.

15 Garcia-Dorado D, Therous P, Tornos P, Sambola A, et al. Previous aspirin use may attenuate the severity of the manifestation of acute ischaemic syndromes. Circulation 1995;92:1743-8.

16 Ginj J van. Low doses of aspirin in stroke prevention. Lancet 1999;353:2172-3.

17 Chinese Acute Stroke Collaborative Group (CAST). Chinese Acute Stroke Collaborative Group: randomised placebo-controlled trial of early aspirin use in 20,000 patients with acute ischaemic stroke. Lancet 1997;349:1641-7.

18 International Stroke Trial Collaborative Group. A randomised trial of aspirin, subcutaneous heparin, both, or neither among 19,435 patients with acute ischaemic stroke. Lancet 1997;349:1569-81.

19 Kmietowicz Z. Aspirin benefits patients with stroke - but only just. $\mathrm{Br}$ Med J 1997;314:1646.

20 Hellemons BSP, Langenberg M, Lodder J, Vermeer F, et al. Primary prevention of arterial thromboembolism in non-rheumatic atrial fibrillation in primary care: randomised controlled trial comparing two intensities of coumarin with aspirin. Br Med J 1999;319:958-64.

21 Anon. Prevention of pulmonary embolism and deep vein thrombosis with low-dose aspirin: Pulmonary Embolism Prevention (PEP) trial. Lancet 2000;355:1295-302.

22 Steering Committee of the Physicians' Health Study Research Group. Final report on the aspirin component of the on-going Physicians' Health Study. N Engl J Med 1989;321:129-35.
23 Peto R, Gray R, Collins R, Wheatley K, et al. Randomised trial of prophylactic daily aspirin in British male doctors. $\mathrm{Br}$ Med $\mathrm{J}$ 1988;296:313-6.

24 Medical Research Council. Thrombosis prevention trial: randomised trial of low-intensity oral anticoagulation with warfarin and low-dose aspirin in the primary prevention of ischaemic heart disease in men at increased risk. Lancet 1998;351:233-41.

25 Juul-Moller S, Edvardsson N, Jahnmatz B, Rosen A, et al. Double blind trial of aspirin in primary prevention of myocardial infarction in patients with stable chronic angina pectoris. Lancet 1992;340:1421-5.

26 The health of the nation: assessing the options: CHD/Stroke. Target effectiveness and cost-effectiveness of interventions to reduce coronary heart disease and stroke mortality. London: Department of Health, 1995.

27 Tofler GH, Brezinski DA, Schafer AI, Czeisler CA, et al. Concurrent morning increase in platelet aggregability and the risk of myocardial infacrtion and sudden cardiac death. N Engl J Med 1987;316:1514-8.

28 Ridker PM, Manson JE, Buring JE, Muller JE, Hennekens CH. Circadian variation of acute myocardial infarction and the effect of low-dose aspirin in a randomised trial of physicians. Circulation 1990;82:897-902.

29 Second International Study of Infarct Survival Group. Randomised trial of intravenous streptokinase, oral aspirin, both or neither among 17,187 cases of suspected acute myocardial infarction: ISIS-2. Lancet 1988;ii:349-60.

30 Berglund U, Wallentin L. Persistent inhibition of platelet function during long-term treatment with $75 \mathrm{mg}$ acetylsalicylic acid daily in men with unstable coronary artery disease. Eur Heart J 1991;12:428-33.

31 Dabaghi SF, Kamat SG, Payne J, Marks GF, et al. Effects of low-dose aspirin on in-vitro platelet aggregation in the early minutes after injestion in normal subjects. Am J Cardiol 1994;74:720-3.

32 Stein B, Fuster V. Role of platelet inhibitor therapy in myocardial infarction. Cardiovasc Drugs Ther 1989;3:797-85.

33 Love BB, Biller J, Gent M. Adverse haematological effects of ticlopidine. Prevention, recognition and management. Drug Safety 1998;19:89-98.

34 CAPRIE Steering Committee. Randomised, blinded, trial of Clopidogrel versus aspirin in patients at risk of ischaemic events. (CAPRIE). Lancet 1996;348:1329-36.

35 Collins R, MacMahon S, Flather M, Baigent C, et al. Clinical effects of anbticoagulant therapy in suspected myocardial infarction: systematic overview of randomised trials. Br Med J 1996;313:652-9.

36 Choussat R, Montalescot G. Blocking platelets more: are we skating on thin ice? Heart 1998;79:5-6.

37 Belsey J. Lipid-lowering in coronary heart disease. Gavel: Evidence based medicine in practice. 1998;1:2-8.

38 Richards M, Meade TW, Peart S, Brennan PJ, Mann AH. Is there any evidence for a protective effect of antithrombotic medication on cognitive function in men at risk of cardiovascular disease? J Neurol Psychiat 1997;62:269-72.

39 Stewart WF, Kawas C, Corrada M, Metter EJ. Risk of Alzheimer's disease and duration of NSAID use. Neurology 1997;48:626-32.

40 Morgan G. Beneficial effects of NSAIDs in the gastrointestinal tract. Eur J Gastroenterol Hepatol 1999;11:393-400.

Address for correspondence: Professor P C Elwood, MRC Building, Llandough Hospital, Penarth CF64 2XW. E-mail: pelwood@doctors.org.uk 